\title{
THE DUAL GROUP OF THE FOURIER-STIELTJES ALGEBRA
}

\author{
BY MARTIN E. WALTER ${ }^{1}$
}

Communicated by Kenneth A. Ross, March 8, 1972

\begin{abstract}
We announce the definition of the dual group, $G_{B(G)}$, of the Fourier-Stieltjes algebra, $B(G)$, of a locally compact group $G$; and we state four main theorems culminating in the result that $G_{B(G)}$ is a locally compact topological group which is topologically isomorphic to $G$. This result establishes an explicit dual relationship between a group and its Fourier-Stieltjes algebra. Moreover, this result extends naturally the notion of Pontriagin duality to the case of noncommutative groups.
\end{abstract}

1. We shall adopt the notation and assume familiarity with the results of [3], [4]. We recall from [3], [4] that each $\phi \in \operatorname{Aut}(B(G))$, the isometric algebra automorphisms of the Banach algebra $B(G)$, can be written in the form

$$
\phi={ }^{t} T_{g}{ }^{t} \psi
$$

where $\left\langle x,{ }^{t} T_{g} b\right\rangle=\langle g x, b\rangle$ and $\langle x, t \psi b\rangle=\langle\psi(x), b\rangle$ for all $b \in B(G)$ and all $x \in G$, with $g \in G$ and $T_{g}: x \in G \mapsto g x \in G$, a left translation of $G$, and $\psi \in \operatorname{AAut}(G)$, the set of all topological automorphisms and/or antiautomorphisms of $G$. The set of all topological automorphisms of $G$ is denoted by $\operatorname{Aut}(G)$. Note that for a given $\phi \in \operatorname{Aut}(B(G))$ decomposition (1) is unique; and recall that superscript ${ }^{t}$ indicates the transpose in the appropriate duality. We let $I_{g}: x \in G \mapsto g^{-1} x g \in G$ denote the inner automorphism of $G$ induced by $g$; and we observe that $T_{g} I_{g}: x \in G \mapsto x g \in G$ is a right translation of $G$.

2. We begin by defining the underlying topological space of (what shall eventually be the dual group of $B(G)) G_{B(G)}$. This requires first the following intrinsic characterization of $A(G)$, the Fourier algebra of $G$, within $B(G)$.

THEOREM 1. The Fourier algebra of $G, A(G)$, is the smallest norm-closed, nonzero ideal in $B(G)$ which is invariant under $\operatorname{Aut}(B(G))$, i.e.,

$$
\begin{aligned}
& A(G)=\bigcap\{\mathscr{I}: \mathscr{I} \text { is a norm-closed, nonzero ideal in } B(G) \\
& \text { such that } \phi(\mathscr{I}) \subset \mathscr{I} \text { for all } \phi \in \operatorname{Aut}(B(G))\} \text {. }
\end{aligned}
$$

REMARK. The burden of the proof rests on the generalized tauberian

AMS 1970 subject classifications. Primary 22D35, 43A40, 46J99; Secondary 22D05, 22D25, 46L25.

Key words and phrases. Topological group, Fourier-Stieltjes algebra, automorphism group, pure positive definite function, spectrum of commutative Banach algebra, duality.

1 The author is partially supported by the National Research Council of Canada. 
Lemma 3.38 of [2]. This theorem thus gives us an alternative definition of $A(G)$ in terms of $B(G)$.

We denote the set of all norm continuous linear functionals on $B(G)$ by $B(G)^{\prime}$; and $\sigma(B(G)$ ), the spectrum (nonzero multiplicative linear functionals) of $B(G)$ is contained in $B(G)^{\prime}$. We take for the underlying topological space of $G_{B(G)}$ the following:

$$
\{x \in \sigma(B(G)): x \text { restricted to } A(G) \text { is not identically zero }\}
$$

endowed with the relativized $\sigma\left(B(G)^{\prime}, B(G)\right)$ topology. We shall refer to this set as the "set of nondegenerate (or regular) characters of $B(G)$." The motivation for selecting this set as the underlying set of $G_{B(G)}$ came from the first remark following Theorem 1 of [3], or the Lemma in the proof of Theorem 1 of [4].

3. We now come to the main results of this announcement. By using the following Theorem 2, we shall be able to endow the nondegenerate characters of $B(G)$ with a group structure identical to that of $G$. Recall first that $P(G)$, the positive cone of $B(G)$, denotes the set of all continuous positive definite functions. We denote by $P(G)_{1}$ the elements in $P(G)$ of norm one, and by $\operatorname{ext}\left(P(G)_{1}\right)$ the extreme points of $P(G)_{1}$.

TheOREM 2. A given $\phi \in \operatorname{Aut}(B(G))$ satisfies

$$
\left\|p-e^{i \theta} \phi p\right\|^{2}+\left\|p+e^{i \theta} \phi p\right\|^{2}=4 \begin{aligned}
& \text { for all real } \theta \text { and } \\
& \text { all } p \in \operatorname{ext}\left(P(G)_{1}\right),
\end{aligned}
$$

if and only if $\phi={ }^{t} T_{g}{ }^{t} \psi_{g}$ where $g \in G$ and $\psi_{g} \in \operatorname{AAut}(G)$ has the property that for each $p \in \operatorname{ext}\left(P(G)_{1}\right),{ }^{t} \psi_{g} p=$ either $p$ or ${ }^{t} I_{g} p$.

REMARK. It is relatively easy to establish that if, e.g., $\phi={ }^{t} T_{g}$ or $\phi={ }^{t} T_{g}{ }^{t} I_{g}$, then $\left\|p-e^{i \theta} \phi p\right\|^{2}+\left\|p+e^{i \theta} \phi p\right\|^{2} \leqq 4$ for all $p \in P(G)_{1}$, with equality holding if $p \in \operatorname{ext}\left(P(G)_{1}\right)$.

REMARK. The key point of the proof is a computation in two-dimensional Hilbert space which establishes the following inequality for $p \in \operatorname{ext}\left(P(G)_{1}\right)$ :

$$
\left\|p-e^{i \theta_{0} t} T_{g}^{t} \psi p\right\|^{2}+\left\|p+e^{i \theta_{0} t} T_{g}^{t} \psi p\right\|^{2} \geqq 4\left(1+\sin x^{\prime} \sin x\right)
$$

where $x^{\prime}, x \in[0, \pi / 2]$ and $\theta_{0}$ real are (judiciously) chosen, depending on $g$ and $\psi$.

Definitions. If $\phi \in \operatorname{Aut}(B(G))$ satisfies (2), we say $\phi$ is a coherent automorphism of $B(G)$. If $\phi_{1}$ and $\phi_{2}$ are coherent and $\phi_{1} \phi_{2}$ and $\phi_{2} \phi_{1}$ are also coherent, we say $\phi_{1}$ and $\phi_{2}$ are compatible automorphisms of $B(G)$.

REMARK. If ${ }^{t} T_{g}{ }^{t} \psi_{g}$ is coherent and $\left({ }^{t} T_{g}{ }^{t} \psi_{g}\right)^{2}$ is coherent, it can be shown that $\psi_{g} \in \operatorname{Aut}(G)$. Now consider the set $S=\left\{\phi \in \operatorname{Aut}(B(G)): \phi\right.$ and $\phi^{2}$ are coherent $\}$. It can then be shown that if ${ }^{t} T_{g}{ }^{t} \psi_{g} \in S$, we have ${ }^{t} \psi_{g} p_{i}=p_{i}$ 
or ${ }^{t} \psi_{g} p_{i}={ }^{t} I_{g} p_{i}, i=1,2$, where $p_{1}, p_{2} \in \operatorname{ext}\left(P(G)_{1}\right)$ are equivalent in the sense that they are coefficients of the same irreducible representation of $G$. We note that $\operatorname{ext}\left(P(G)_{1}\right)$ can intrinsically be partitioned into equivalence classes (induced by aforementioned equivalence relation) with knowledge of $B(G)$ only.

THEOREM 3. Let ${ }^{t} T_{g}{ }^{t} \psi_{g},{ }^{t} T_{h}{ }^{t} \psi_{h} \in S$ be compatible; then either

(1) $\psi_{g}(h)=h$ and $\psi_{h}(g)=g$ or

(2) $\psi_{g}(h)=I_{g}(h)$ and $\psi_{h}(g)=I_{h}(g)$.

REMARK. The Jordan product of automorphisms and the linear independence of the nondegenerate characters of $B(G)$ are used in the proof of Theorem 3. Note that if for a fixed ${ }^{t} T_{g}{ }^{t} \psi_{g}$ there is a ${ }^{t} T_{h}{ }^{t} \psi_{h}$ compatible with ${ }^{t} T_{g}{ }^{t} \psi_{g}$ (for every nondegenerate character $h$ ), then $\psi_{g}=$ either $I_{g}$ or the identity automorphism.

We are now prepared to define the group multiplication for $G_{B(G)}$. First we observe that there is a natural surjection of $\operatorname{Aut}(B(G))$ onto the set $G_{B(G)}$, viz., \# : $\phi={ }^{t} T_{g}{ }^{t} \psi \in \operatorname{Aut}(B(G)) \mapsto\left({ }^{t} T_{g}^{t} \psi\right)_{\#} \equiv e \circ^{t} T_{g}^{t} \psi$, where $e$ is that unique nondegenerate character of $B(G)$ satisfying $\langle e, p\rangle=\|p\|$ for all $p \in P(G)$ (or alternatively, for all $p \in \operatorname{ext}\left(P(G)_{1}\right)$ ).

Note. $\left\langle e \circ^{t} T_{g}{ }^{t} \psi, b\right\rangle=\left\langle e,{ }^{t} T_{g}{ }^{t} \psi(b)\right\rangle$ for $b \in B(G)$.

Now extract from $\operatorname{Aut}(B(G))$ a subgroup $M$ of coherent, pairwise compatible automorphisms such that the \#-map restricted to $M$ is still a surjection. (Such an $M$ exists and is isomorphic to $G$ !) Given $x, y$ two nondegenerate characters, it can be shown that if $\left({ }^{t} T_{g}{ }^{t} \psi_{g}\right)_{\#}=x$ and $\left({ }^{t} T_{h}{ }^{t} \psi_{h}\right)_{\#}=y$, defining $x y=\left({ }^{t} T_{g}{ }^{t} \psi_{g}{ }^{t} T_{h}{ }^{t} \psi_{h}\right)_{\#}$ gives a group product on these characters, if ${ }^{t} T_{g}{ }^{t} \psi_{g}$ and ${ }^{t} T_{h}{ }^{t} \psi_{h} \in M$. We now have

THEOREM 4. The topological space of nondegenerate characters of the Fourier-Stieltjes algebra, $B(G)$, of a locally compact group $G$, endowed with the group structure it naturally inherits from $\operatorname{Aut}(B(G))$ (cf. preceding paragraph) is a locally compact topological group, $G_{B(G)}$, which we call the dual group of $B(G)$. This group $G_{B(G)}$ is topologically isomorphic to $G$.

REMARK. To clarify the motivation for our "proofs", we have in this announcement adopted the (perhaps inelegant) point of view that we were given $B(G)$, the Fourier-Stieltjes algebra of some group $G$, that we then "forgot" the structure of $G$ and that we then reconstructed the topological and algebraic structure of $G$ using whatever properties of $B(G)$ that were necessary for the task. Having noted what properties of $B(G)$ were necessary, we are in a position to abstractly (and perhaps more elegantly) characterize a Fourier-Stieltjes algebra, define its dual group, construct in turn its Fourier-Stieltjes algebra, etc.; hopefully we will obtain in the process an analogue of Pontriagin's duality theorem. 
For detailed proofs of the facts only briefly sketched herein as well as some peripheral material not mentioned, we refer the reader to [5]. The author would once again like to thank B. Russo and M. Takesaki for introducing the Fourier algebra to him.

\section{REFERENCES}

1. C. A. Akemann and M. E. Walter, Non-abelian Pontriagin duality, Duke Math. J. (to appear).

2. P. Eymard, L'algèbre de Fourier d'un groupe localement compact, Bull. Soc. Math. France 92 (1964), 181-236. MR 37 \# 4208.

3. M. E. Walter, Group duality and isomorphisms of Fourier and Fourier-Stieltjes algebras from a $W^{*}$-algebra point of view, Bull. Amer. Math. Soc. 76 (1970), 1321-1325.

4. $-W^{*}$-algebras and non-abelian harmonic analysis, J. Functional Analysis (to appear).

5. - A duality between locally compact groups and certain Banach algebras, Queen's Univ. (preprint in preparation).

Department of Mathematics, Quben's University, Kingston, Ontario, Canada 